\title{
Photonic molecules modes in resonantly coupled spherical microcavities with semiconductor nanocrystals
}

\author{
Y.P. Rakovich ${ }^{* a}$, M. Gerlach ${ }^{\mathrm{a}}$, A.L. Bradley ${ }^{\mathrm{a}}$, J.F. Donegan ${ }^{\mathrm{a}}$, T.Connolly ${ }^{\mathrm{b}}$, J.J. \\ Boland $^{\mathrm{b}}$, N.Gaponik ${ }^{\mathrm{c}}$ and A. L. Rogach ${ }^{\mathrm{d}}$ \\ ${ }^{\mathrm{a}}$ Semiconductor Photonics Group, Department of Physics, Trinity College Dublin, \\ Dublin 2, Ireland \\ ${ }^{\mathrm{b}}$ Department of Chemistry, Trinity College Dublin, Dublin 2, Ireland \\ ${ }^{\mathrm{c}}$ Institute of Physical Chemistry, University of Hamburg, 20146 Hamburg, Germany \\ ${ }^{\mathrm{d}}$ Photonics and Optoelectronics Group, Physics Department and CeNS, \\ Ludwig-Maximilians Universität München, 80799 Munich, Germany
}

\begin{abstract}
ABSRACT
We present a detailed study of the localized coupled-cavity modes in a photonic molecule formed from two dielectric spherical microcavities with CdTe nanocrystals, which show a multi-peak narrowband modal structure resulting from lifting of the mode degeneracy with respect to the azimuthal quantum number. The feasibility of photonic molecules as the basis for a multi-channel, wavelength-tunable optical delay device is analysed.
\end{abstract}

Keywords: coupled microcavities, semiconductor nanocrystals , whispering gallery modes, mode degeneracy, optical delay

\section{INTRODUCTION}

There is currently great interest in design and fabrication of optical structures for manipulation of light on micrometer-length scale. Much of this attention has been focused on photonic band-gap structures or two-dimensional photonic wires due to the possibility of optical interconnection ${ }^{1}$. Closely related to these developments is the intensive work on the modification of photon modes in spherical microcavities, which has been of great interest both for studies of fundamental optical properties and for the potential applications ${ }^{2}$.

Extending the ideas of the linear combination of atomic orbitals method to the classical wave case, it was recently suggested that Mie resonances of single spherical microcavity play the same role as the atomic orbitals in the electronic case and the spatial distributions of wispering gallery modes (WGM) can be described by analogy with the orbitals in a hydrogen atom ${ }^{3}$. In the absence of gain, the WGM resonances can be characterized by a mode number $n$ (angular quantum number), mode order $l$ (radial quantum number), and azimuthal mode number $m$ (azimuthal quantum number). The value of $n$ is proportional to the circumference divided by the wavelength of the light propagating within the microsphere, the mode order $l$ indicates the number of maxima in the radial distribution of the internal electric field, and the azimuthal mode number $m$ gives the orientation of the WGMs orbital plane. WGMs in a single sphere are degenerate with respect to $m$ because of the spherical symmetry.

The electro-magnetic fields confined in microsphere are given by ${ }^{4}$

$\mathbf{E}_{T E}=j_{n}(n k r) \mathbf{X}_{n m}(\theta, \phi)$

for modes having no radial components of the electric field (transverse electric or TE modes) and

$\mathbf{E}_{T M}=\nabla \times j_{n}(n k r) \mathbf{X}_{n m}(\theta, \phi)$,

\footnotetext{
*Yury.Rakovich@tcd.ie; phone +353-1-608-2193; fax 353-1-671-1759
} 
for the transverse magnetic (TM) modes (no radial component of the magnetic field).

Here $X_{n m}(\theta, \phi)$ are the vector spherical harmonics given in spherical polar, $j_{n}\left(n_{r} k r\right)$ represents the spherical Bessel function, where $k=2 \pi / \lambda$ is the wavenumber, $n_{r}$ is the refractive index of the sphere and $\lambda$ is the free space wavelength.

On the other hand, the wave functions $\Psi_{s l m}$ for the electron confined in the hydrogen atom are given by ${ }^{5}$

$$
\Psi_{s l m}=R_{s l}(r) \mathrm{Y}_{l m}(\theta, \phi),
$$

where $R_{s l}(r)$ is known as the Laguerre polynomials. The eigenfunctions (1) and (3) are very similar and their spatial distributions are characterized by the three integer $s, l$ and $m$ (for hydrogen atom) and $n, l$ and $m$ (for microsphere), which correspond to total angular, radial and the azimuthal quantum numbers, respectively. Based on all above similarities this approach has enabled small dielectric spheres to be considered as "photonic atoms" 3, 6. However, unlike energy states of electron in the atom, photonic states in spherical microcavities are not localized due to finite storage time $(\tau)$ of photons in the resonant mode. This "photon lifetime" is controlled by quality factor of WGM Q and therefore can be limited by diffractive losses, absorption, gain, shape deformation or refractive index inhomogeneity ${ }^{4}$. As a result, the resonant internal field of a spherical cavity is not completely confined to the interior of the microparticle. Depending on the size of the microsphere, the evanescent field can extend into the surroundings up to a couple of micrometers. It was recently recognized that the partial delocalization of Mie resonance states is of great importance because it suggests a possibility for coherent coupling between WGMs of two adjacent spherical particles with closely matched sizes. Such a system of coherently coupled photonic atoms may be called a "photonic molecule" (PM) ${ }^{7}$ and can be employed in order to manipulate photons in the micrometer length scale. In analogy to the formation of molecular electronic orbits, the tight binding approximation provides two combinations for the electromagnetic field in a system of interacting microspheres: bonding $(\mathrm{BN})$ and antibonding $(\mathrm{ABN})$ states ${ }^{7-10}$. Experimentally, the coupling of the photon modes of individual microspheres in the PM can cause a narrow resonance of a photonic atom to split into two modes of lower $Q$-factor ${ }^{8}$. This phenomenon has been demonstrated in a system of two square, photonic dots coupled by a narrow channel ${ }^{7}$ in a dye-stained bisphere system ${ }^{9,11,12}$, in photonic dots doped by CdSe nanocrystals (NCs) ${ }^{13}$ and in chains of polymer-blend microparticles ${ }^{14}$. However recent theoretical considerations ${ }^{10}$ and experimental studies ${ }^{15,16}$ reveal complex internal distribution of density of photonic states of PM originating from lifting of degeneracy of PM modes with respect to the azimuthal index.

\section{EXPERIMENT}

In this paper we present a detailed study of the localized coupled-cavity modes in a PM formed from two dielectric spherical microcavities with CdTe NCs. Aqueous dispersions of melamineformaldehyde (MF) microspheres, of $3 \pm 0.05 \mu \mathrm{m}$ in diameter, were combined with luminescent $\mathrm{CdTe}$ NCs using a layer-by-layer deposition technique ${ }^{17}$. The colloidal solution of CdTe NCs, with a PL maximum at $563 \mathrm{~nm}(2.9 \mathrm{~nm}$ radius $)$ and a PL quantum efficiency of $\sim 15 \%$ at room temperature, was used for coating MF microspheres. The PL spectra from PM were recorded using a RENISHAW micro-PL system equipped with a positioning stage and an $\operatorname{Ar}^{+}$laser $(\lambda=488 \mathrm{~nm}, 1.0 \mathrm{~mW}$ power $)$.

The small size of the MF spheres was dictated by specific requirements for the optimal excitation conditions like matching the laser wavelength to one of the WGM frequencies and achieving good correlation between the WGM and the laser linewidths. The wide separation of WGMs in spheres of this size allows us to avoid intricate band mixing in the PM. Moreover, the quality factor of $3 \mu \mathrm{m}$ spherical particles forming the PM $\left(\mathrm{Q} \sim 10^{3}\right)$ provides better mode coupling than would be the case with larger microspheres 9 . We proceed from the assumption that a larger coupling parameter can be of crucial importance in the interaction between electromagnetic fields of two adjacent spheres, in order to experimentally study the fine spectral structure of coupled WGMs. 


\section{RESULTS AND DISCUSSION}

To investigate the photon modes in interacting spherical microcavities we first measured PL spectra scanning a sample along the longitudinal axis of the PM (Scheme 1., Fig.1). The observed spectral structure originates from coupling of electronic transitions in NCs to the photon modes of the microsphere, with PL peaks corresponding to the resonant frequencies of WGMs with transverse electric (TE) and transverse magnetic (TM) polarizations. In Fig. 1 one can clearly see a number of narrow peaks arising from TE and TM WGM of the individual spheres. The pronounced double structure, with the intensity distribution dependent on the excitation position, is just a result of superposition of the uncoupled WGMs of individual microspheres.

For spherically symmetric microparticles the extinction cross-section is derivable from Lorenz-Mie theory ${ }^{18}$ :

$$
C_{e x t}=\frac{2 \pi}{k^{2}} \operatorname{Re} \sum_{n=1}^{\infty}(2 n+1)\left(b_{n}\left(x, n_{r}\right)+a_{n}\left(x, n_{r}\right)\right),
$$

where $x=2 \pi r / \lambda$ is the size parameter, $r$ is the radius of microsphere and the Mie scattering partial wave amplitudes $\mathrm{a}_{\mathrm{n}}\left(\mathrm{x}, \mathrm{n}_{\mathrm{r}}\right)$ and $\mathrm{b}_{\mathrm{n}}\left(\mathrm{x}, \mathrm{n}_{\mathrm{r}}\right)$ can be expressed in the form ${ }^{19}$

$a_{n}\left(x, n_{r}\right)=\frac{A_{n}\left(x, n_{r}\right)}{A_{n}\left(x, n_{r}\right)+i C_{n}\left(x, n_{r}\right)} \quad$ and $\quad b_{n}\left(x, n_{r}\right)=\frac{B_{n}\left(x, n_{r}\right)}{B_{n}\left(x, n_{r}\right)+i D_{n}\left(x, n_{r}\right)}$

The pairs of functions $A_{n}, C_{n}$ and $B_{n}, D_{n}$ are determined by the Mie scattering problem ${ }^{20}$ and resonance structure in scattering spectra can be expected as the real part of $a_{n}$ or $b_{n}$ reaches its maximum value of 1 and the imaginary part is passing through 0 from the positive to the negative side. In other words the resonances in the Mie scattering characteristics occur when $C_{n}=0$ or $D_{n}=0$, which gives the the following mathematical condition for a resonance ${ }^{21}$ :

$$
n_{r} \psi_{n}(x) \psi_{n}^{\prime}\left(n_{r} x\right)-\psi_{n}\left(n_{r} x\right) \psi_{n}^{\prime}(x)=0
$$

or

$$
n_{r} \chi_{n}(x) \psi_{n}^{\prime}\left(n_{r} x\right)-\psi_{n}\left(n_{r} x\right) \chi_{n}^{\prime}(x)=0
$$

Note, that for given $n$ and $m$, these equations have infinitely many solutions at discrete values of $x$ for TM and TE WGMs respectively. The Riccati-Bessel functions of the first and second kind can be introduced as:

$\psi_{n}(z)=z j_{n}(z)=\sqrt{\frac{\pi z}{2}} J{ }_{n+\frac{1}{2}}(z) ; \quad$ and $\quad \chi_{n}(z)=z y_{n}(z)=\sqrt{\frac{\pi z}{2}} Y_{n+\frac{1}{2}}(z)$

where $J(z)$ and $Y(z)$ are the cylindrical Bessel functions of the first and second kind respectively. The use of Bessel functions for systems with cylindrical symmetry together with reccurence relation

$\psi_{n}^{\prime}(z)=-\frac{n}{z} \psi_{n}(z)+\psi_{n-1}(z)$ enables to reduce the equations (6)-(7) to the form convenient for practical calculation of position of WGMs. Thus for real refractive index conditions for TM and TE resonances can be taken in the form:

$\frac{1}{x}\left(\frac{n}{n_{r}}-n_{r} n\right) J_{n+1 / 2}(x) J_{n+1 / 2}\left(n_{r} x\right)+n_{r} J_{n+1 / 2}\left(n_{r} x\right) J_{n-1 / 2}(x)-J_{n+1 / 2}(x) J_{n-1 / 2}\left(n_{r} x\right)=0,(9)$ 
$n_{r} Y_{n+1 / 2}(x) J_{n-1 / 2}\left(n_{r} x\right)-J_{n+1 / 2}\left(n_{r} x\right) Y_{n-1 / 2}(x)=0$.

These conditions are a transcendental equations, which can be solved for the size parameter $\mathrm{x}$ (position of a resonance) for given values of refractive index $m$ and for given angular quantum number $n$. Thus comparing calculated results with the spectral positions of the WGM in the experimental PL spectra we can identify the indexes $n$ and $l$ for each mode and estimate the size of the sphere. The algorithm of the mode assignment can be as follows. 1) The resonant wavelengths corresponding WGM resonances $\lambda_{i}^{\exp }(\mathrm{i}=1,2 \ldots, \mathrm{N})$ are determined from a PL spectrum of single sphere. 2) We assume approximate value microsphere radius based on technical specification within distribution of the sizes. 3) Theoretical resonance positions $\lambda_{i}^{\text {theor }}$ are then calculated using eqs. (8) and (9). 4) Two lists are compared and for each value of $\lambda_{i}^{\text {exp }}$ the closest value $\lambda_{i}^{\text {theor }}$ is suggested and difference between them $\Delta_{\mathrm{i}}$ is calculated. 5) Taking into account spectral resolution $\Delta$ the correlation $S=\frac{1}{N} \sum_{1}^{N}\left(1+\Delta_{i} / \Delta\right)^{-1}$ is then maximized by adjusting only one fitting parameter, namely size of microsphere.

Figure 1 shows a result of WGM identification by this algorithm. The shift in position of the $T E_{22}^{1}, T E_{23}^{1}, T M_{21}^{1}$ and $T M_{22}^{1}$ modes of single microspheres, which can be clearly seen in Fig. 1 is a result of a difference in the size of the two microspheres of only $11 \mathrm{~nm}$ as follows from our calculations based on Mie theory. When the excitation is provided at the contact point between microspheres (Fig.1,b), the excited and emitted light propagate efficiently along the longitudinal axis of the bisphere and the contribution of both microcavities can be clearly seen in the PL spectra at discrete WGM wavelengths. This contribution of PL signal from individual microcavities can be switched by changing excitation position from the left to the right rim of PM (Fig. 1, a, c). The above experiments have involved excitation beam incidence perpendicular to the longitudinal axis of the PM. For a given excitation and detection configuration we did not observe any new peaks in the PL spectra of the PM as compared to that of single spheres which is indication of weak intermode coupling. The lack of intermode coupling in this configuration (Scheme 1) is not surprising because the coupling between electro-magnetic fields of the spheres is expected to be minimum when the excitation and detection are set perpendicular to the axis of the $\mathrm{PM}^{9}$. However, the observed redistribution of intensity between the components of WGM double structure clearly demonstrates the waveguiding of the light along the PM and the possibility of wavelength switching in the PM depending on excitation-detection geometry.
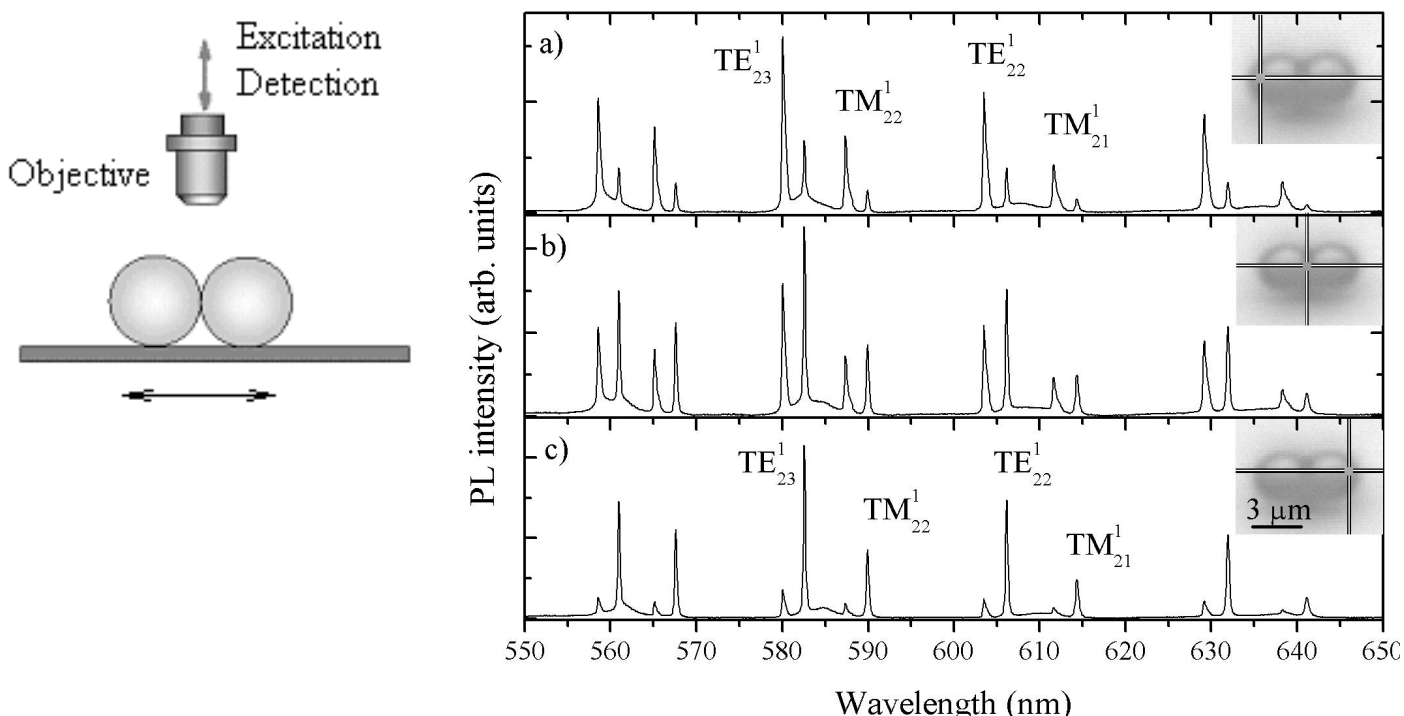

Scheme 1: Experimental geometry for scanning PM along the longitudinal axis.

Figure 1: PL spectra of a PM with excitation and detection at three different positions along its longitudinal axis. Insets: microscope images of the PM, with the cross-hairs indicating the excitation-detection position. 
Theoretical considerations ${ }^{8,10}$ show that the intersphere coupling is expected to be maximum for the pair of modes whose orbitals include the contact point between microspheres and lie in the same plane 9 . Taking this into account, the PL intensity of the coupled modes is anticipated to be maximum in the direction parallel to the PM axis and the signal from the coupled intersphere modes should be more pronounced in the parallel configuration than in the perpendicular one.

In order to control the alignment of the spheres constituting the PM we utilized a polystyrene substrate containing a three-dimensionally ordered array of pores of $\sim 5 \mu \mathrm{m}$ in size prepared through a thermocapillary convection ${ }^{22}$. The ordered structures are formed by evaporating solutions of polystyrene in a volatile solvent, in the presence of moisture with forced airflow across the solution surface. A hexagonally packed array of holes (microwells) of 3-5 $\mu \mathrm{m}$ depth then forms on the surface of the polymer (Fig.2). Only one pair of the $3 \mu \mathrm{m}$ microspheres can be accommodated within each microwell, and the axis of the PM is close to the surface normal (Scheme 2).

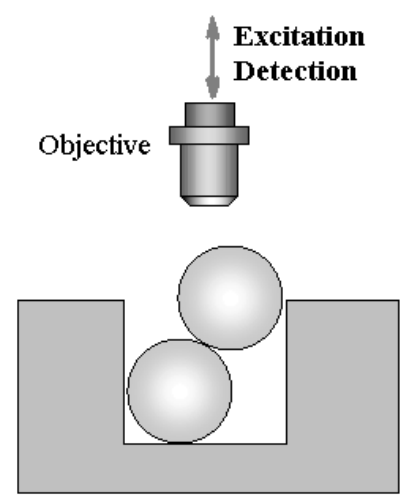

Scheme 2: Experimental geometry for off-axis or parallel excitation and detection geometry.

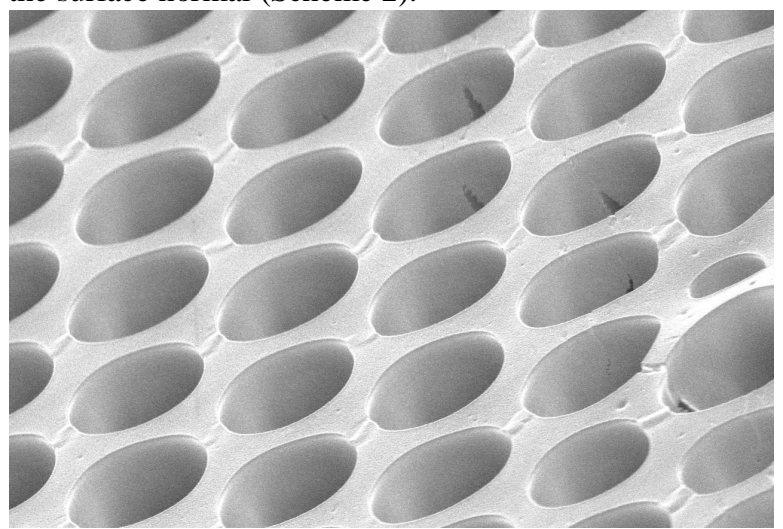

Figure 2: Polystyrene films with hexagonally ordered arrays of air holes with diameters of about $5 \mu \mathrm{m}$.

Figure 3 shows the PL spectrum of a PM accommodated in a microwell and the spectra of the individual microspheres prior to being manipulated into the microwell. Pronounced doublet structure in the spectrum presented in Fig. 3(a), coinciding with the spectral positions of WGM of individual microspheres (Fig. 3(b)), is a result of the overlap of uncoupled modes of the two spheres. The shift in position of the WGM, which can be clearly seen in Figs. 3(a) and 3(b), is a result of a difference in the size of the two microspheres of only $25 \mathrm{~nm}$. However, in contrast to the spectrum in Fig. 3(b), this doublet is accompanied by two extra relatively broad peaks (indicated by arrows in Fig. 3(a)), which are indicative of strong mode coupling in the PM. The appearance of these two satellites can be interpreted as a result of the formation of $\mathrm{BN}$ and $\mathrm{ABN}$ orbitals in the $\mathrm{PM}^{7}$ with the $\mathrm{ABN}$ peak observed at lower wavelength than the $\mathrm{BN}$ one. In terms of cooperative scattering theory ${ }^{8}$, the observed satellites originate from the removal of the WGM degeneracy with respect to the $m$ index. The line shape of the satellite lines reflects the energy distribution among the coupled modes, because modes with different combinations of $m$ can contribute to the PL spectra. The observation of a broader $\mathrm{ABN}$ peak, relative to the $\mathrm{BN}$ peak, reflects the decrease of the quality factor of the PM when compared with that of a single sphere presumably due to the interaction with more dissipative modes of lower $l^{9}$. The deconvolution of the lineshape of resonances belonging to $T E_{22}^{1}$ WGM (Figure $3 \mathrm{a}, \mathrm{b}$ ) using Lorentian functions show that the quality factors of PM peaks are $\sim 8$ times smaller than Qfactors of noninteracting microspheres.

The high PL efficiency of CdTe NCs and the coupling of electronic transitions of NCs to the resonances of the PM allows us to detect the $\mathrm{BN}$ and $\mathrm{ABN}$ branches in a wide spectral region from 525 to $725 \mathrm{~nm}$, corresponding to the full spectral range of the NC emission. This allows the possibility of estimating the magnitude of spectral spacing between bonding and antibonding branches as a function of angular mode number for comparison with theoretical calculations (Fig.4).

In order to calculate the BN/ABN splitting we adopted the maximum term approximation (MTA) of the single-mode tight-binding (SMTB) method ${ }^{10}$. For simplicity in our simulations we have considered only the case of identical spheres with WGM having radial quantum number $l=1$, taking into account only the interaction between WGM of the same $n$. 


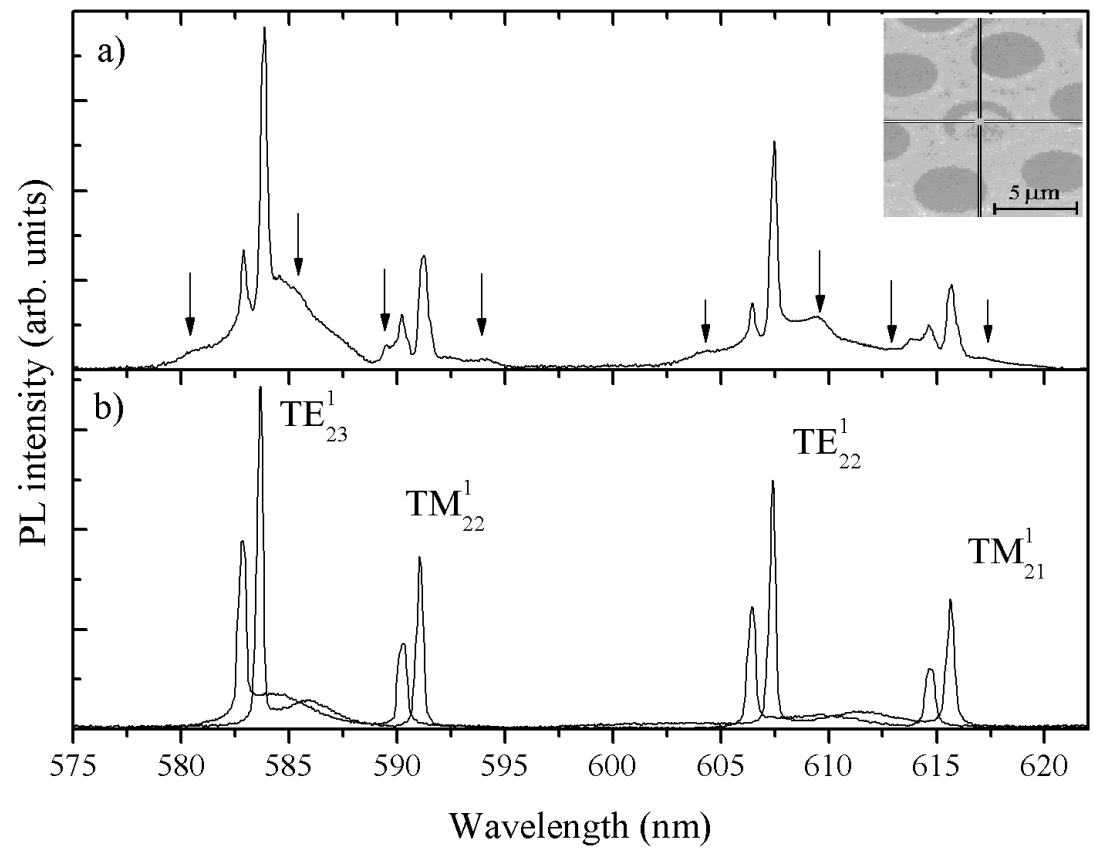

Figure 3: (a) The PL spectrum of a PM accommodated in a microwell. Arrows indicate the coupled modes. (b) The PL spectra of noninteracting microspheres. Inset: microscope image of the PM in the microwell. The dark cross indicates the excitation position.

For a given WGM polarization, the value of the splitting between the $\mathrm{BN}$ and $\mathrm{ABN}$ modes of PM can be obtained from

$$
\Delta x_{m}=2 \Gamma_{n}\left|A_{n, m}\left(x_{n}\right)\right|
$$

where $x_{n}=2 \pi R / \lambda_{n}$ is the size parameter of a resonance with mode number $n, \lambda_{n}$ is the corresponding resonant wavelength, $\Gamma_{\mathrm{n}}$ is the width of mode $n$ in a single sphere, which can be calculated within Mietheory. For a given radius of single spheres $a$, the coefficient $A_{\mathrm{n}, \mathrm{m}}\left(\mathrm{x}_{\mathrm{n}}\right)$ can be calculated as:

$$
A_{n, m}\left(x_{n}\right)=-2 n(-1)^{n+m} h_{2 n}^{(1)}\left(k_{0} Z\right) \times \sqrt{\frac{2 n}{\pi(n+m)(n-m)}} \times \frac{n^{2 n}}{(n+m)^{n+m}(n-m)^{n-m}},
$$

where $\mathrm{k}_{0}=\mathrm{x}_{\mathrm{n}} / \mathrm{a}$ is the wavenumber, $\mathrm{Z}$ is the characteristic length ${ }^{10}$, and the spherical Hankel function of the first can be estimated from

$$
h_{2 n}^{(1)}\left(k_{0} Z\right) \cong-i \frac{\exp [(2 n+1 / 2)(\alpha-\tanh \alpha)]}{(n+1 / 2) \sqrt{\sec h \alpha \tanh \alpha}} ;
$$

Here, $\alpha$ is defined by $\mathrm{k}_{0} \mathrm{Z}=(2 \mathrm{n}+1 / 2) / \cosh \alpha$. The positions of the $m$-resonances were estimated as $\mathrm{x}_{\mathrm{m}}=$ $\mathrm{x} \pm \Delta \mathrm{x}_{\mathrm{m}} / 2$.

Applying these equations we can see that the modeled data, presented in Fig. 4 displays behavior which is in good agreement with experimental data. From micro-PL spectra of the PM we have found a decrease in splitting between $\mathrm{BN}$ and $\mathrm{ABN}$ branches value with increasing $n$, with TE modes splitting being always higher than that of TM modes. Remarkably, the theory predicts the same behavior. Although the maximum term approximation overestimates the splitting (Fig. 4), it is clear 
that this approach provides a good qualitative guide for the analysis of a variety of phenomena observed in PM.

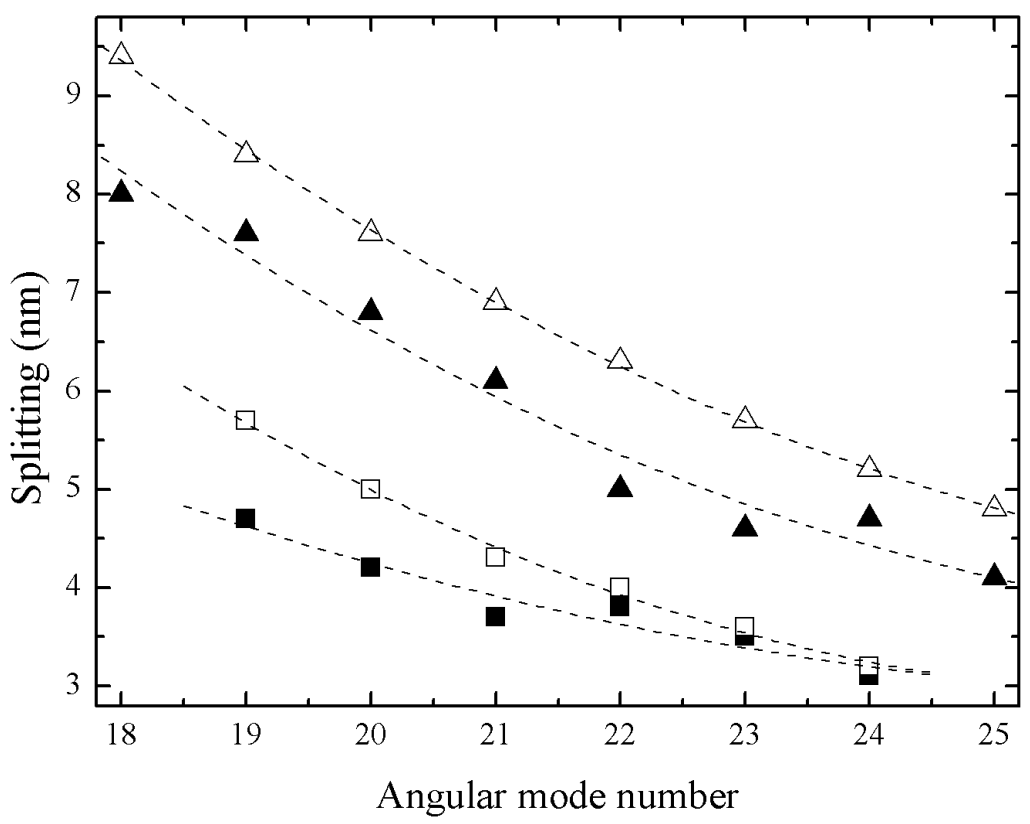

Figure 4: Magnitude of spectral spacing between bonding and antibonding branches estimated from micro-PL spectra of PM as a function of angular mode number for TE (solid up-triangles) and TM (solid squares) modes. Open symbols show the result of theoretical calculation. The dashed lines are a guide to the eye.

It is worth noting that observed disagreement between calculated and experimental values for the PM mode splitting may also have its origin in the fact that the magnitudes of splitting were estimated as a spectral distance between maxima of $\mathrm{ABN}$ and $\mathrm{BN}$ peaks. However detailed consideration of coherent mode coupling in PMs using SMTB approach shows that in fact the BN and $\mathrm{ABN}$ branches consist of a number of very narrow peaks, which are due to the presence of $m \neq \pm 1$ components ${ }^{10}$. The total number of these sharp peaks, originating from a certain mode $n$, is governed by the actual degeneracy of the Mie resonances which in the approach of the normal mode concept is $n$ +1 . (Due to the dependence of the mode coupling on the orbital plane orientation, the interaction is limited between modes of only the same $m$, no degeneracy is removed between $m$ and $-m$ and the new degeneracy of PM modes is now $n+1$ with $m$ running from 0 to $n){ }^{10}$. In our calculations within the SMTB model we estimated the splitting as a spectral distance between outer peaks assigned to modes with $m= \pm 1$, which do not correspond to the maximum of envelope of PM modes and therefore this may cause discrepancy between estimated and observed values of the mode splitting. The fine structure of $\mathrm{BN}$ and $\mathrm{ABN}$ modes of PM is of great interest to the experimentalists because it suggests a manifold of applications particularly in the field of information processing ${ }^{16}$. However in order to observe this phenomenon two conditions should be met. First, the spacing between m-resonances forming BN and ABN modes of PM strongly depends on the angle of incidence of electromagnetic wave $\theta^{9-10,16}$. For $\theta=0^{\mathrm{O}}$, when the incident light propagates parallel to the longitudinal axis of the PM, the incident wave can preferentially excite $m= \pm 1$ modes. The interaction with other dissipative modes of lower $l$ causes the broadening of $\mathrm{BN}$ and $\mathrm{ABN}$ peaks and decreases the $\mathrm{Q}$-factor relative to that of single spheres, as was observed in a number of papers ${ }^{8-9,15-16}$. In the perpendicular configuration, when $\theta=$ $90^{\circ}$, the intermode coupling and spacing between $m$-resonances are expected to be minimal. In other words, in the case of perpendicular orientation of the PM with respect to excitation, all m-modes would merge into broad $\mathrm{BN}$ and $\mathrm{ABN}$ features and their fine structure would not be easily recognized. A compromise can be reached only for off-axis excitation with $0^{\circ}<\theta<90^{\circ}$. Secondly, in order to reveal the $m \neq \pm 1$ components interacting cavities should have not only similar size, but also similar 
Q-factors. If the resonances of the two cavities are of greatly different width, the coherent coupling is disturbed (at least to some extent) and therefore fine structure of PM modes cannot be detected.

One can see from the inset in Fig. 3a that excitation and detection in this case was provided at the centre of the upper sphere forming the PM i.e. along the PM axis. In order to reveal the $m \neq \pm 1$ components in the PL spectra of the MF/CdTe PM we have investigated WGM structure at different excitation (and detection) positions across the upper sphere.

Figure 5a shows micro-PL spectra of a PM formed by two almost identical microspheres (sample B) with sizes of $3.0168 \mu \mathrm{m}$ and $3.0189 \mu \mathrm{m}$ measured for excitation (and signal collection) at the right edge of the upper microsphere (inset, Fig.5a). The presented PL spectra clearly reveal major features unique to strong coherent coupling between the photonic states of the two microspheres forming the PM. One can clearly see a number of narrow peaks grouping on both sides arising from TE and TM resonances of the individual spheres and forming $\mathrm{BN}$ and $\mathrm{ABN}$ modes of PM. The origin of this fine structure of $\mathrm{BN}$ and $\mathrm{ABN}$ modes lies in the lifting of the mode degeneracy in the PM ${ }^{10,16}$ and therefore this multi peak structure would not be radically altered in scattering spectra or even in lasing, or stimulated Raman scattering in the same way that in a semiconductor laser the lasing does not alter the cavity resonances.

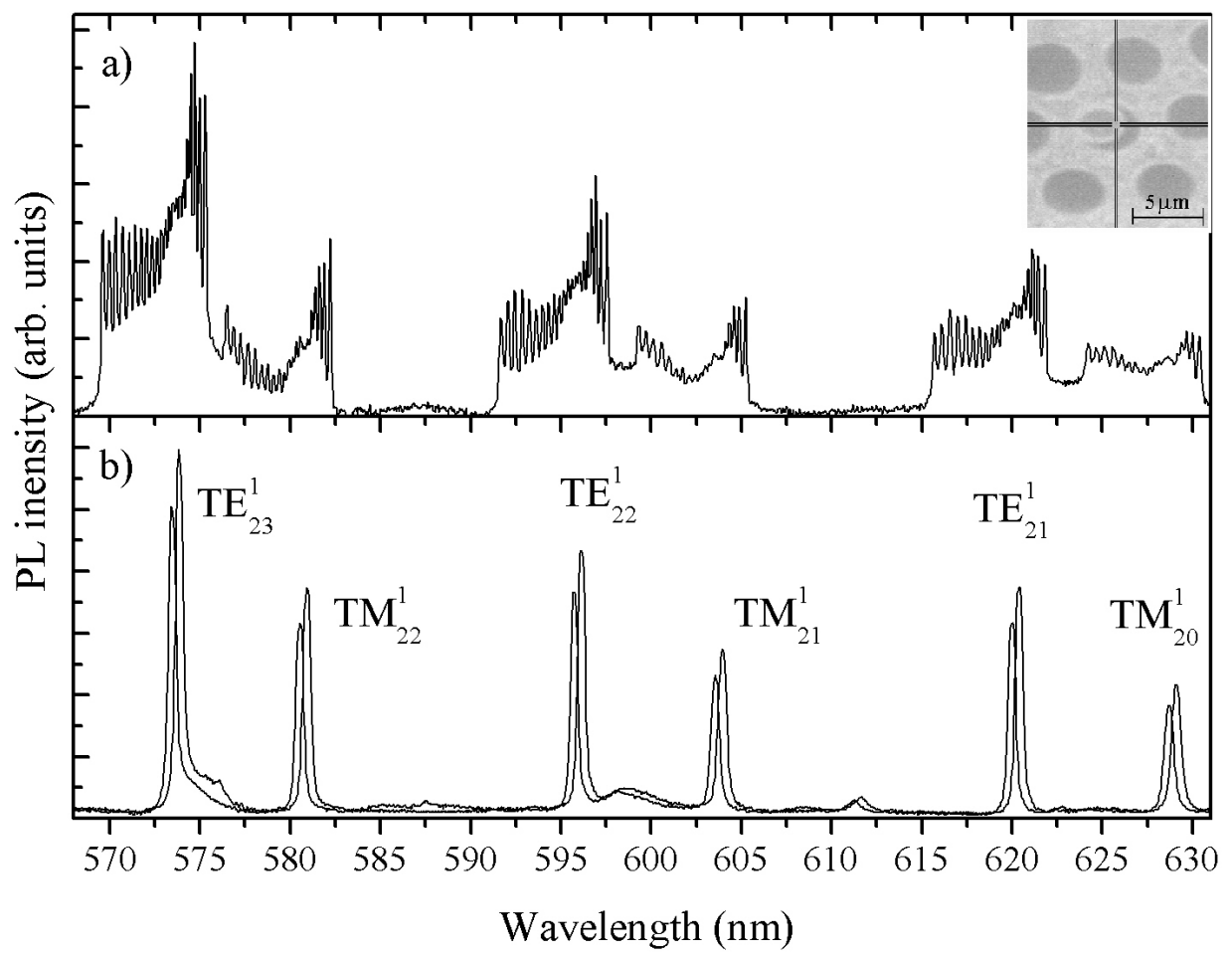

Figure 5: (a) PL spectra of the PM formed by two almost identical microspheres with off-axis excitation and detection. (b) The PL spectra of noninteracting microspheres. Inset: microscope image of the PM in the microwell. The dark cross indicates the excitation position.

It turns out that the number of experimentally resolved peaks increases with angular mode number both for TE and TM modes, and is very close to the $n$ value, although never in excess of $n$. Indeed we have observed 19 peaks in the spectral region of $T E_{20}^{1}\left(19\right.$ peaks for $\left.T M_{20}^{1}\right), 21$ peaks around the $T E_{21}^{1}$ resonance (20 peaks for $T M_{21}^{1}$ ), 21 peaks in the region of the $T E_{22}^{1}$ resonance (21 peaks for $T M_{22}^{1}$ ), 22 peaks for $T E_{23}^{1}$ (22 peaks for $T M_{23}^{1}$ ) and 23 peaks around the $T E_{24}^{1}$ resonance. Note that the spectral region occupied by a set of these narrow resonances is much wider than linewidth of WGMs of non-interacting microspheres (Fig.5). 
The deconvolution of the lineshape of the $m$-resonances of the PM using Lorentian functions shows that $m$-resonances of the $\mathrm{BN}$ branch are always sharper than that of $\mathrm{ABN}$ one, providing a higher quality factor $\mathrm{Q}$ value for these modes and therefore a higher photon lifetime in the resonant modes $\tau^{16}$. However, the most remarkable experimental fact is that the $\mathrm{Q}$ factor of the $m$-resonances in the spectra of the PM exceeds the $\mathrm{Q}$ value of single noninteracting microspheres, suggesting the respective modification of photon lifetime in PM $\left(\tau_{\mathrm{PM}}\right)$ relative to photon storage time in single spherical microcavities before contact $\left(\tau_{\mathrm{ss}}\right)$. These two facts along with estimated value of BN/ABN splitting $(\sim 5-7 \mathrm{~nm})$ implies the possibility for development of a new PM-based photonic device such as an optical delay line with controllable spectral and temporal tunability.

Figure 6 shows spectral distribution of the ratio between photon lifetime of $m$-modes of PM and photon storage time in WGMs of single sphere calculated from corresponding values of mode linewidth in the region of $T E_{21}^{1}$ and $T M_{20}^{1}$ resonances demonstrating a threefold photon storage enhancement. It is evident from Fig. 6, that the interaction between spherical microcavities results in periodic group delay spectra with peaks occurring at each of the $m$-resonant frequencies with bigger delay times for higher $m$-values, which implies that the spectral components near these $m$-resonance spend more time traveling within the PM.

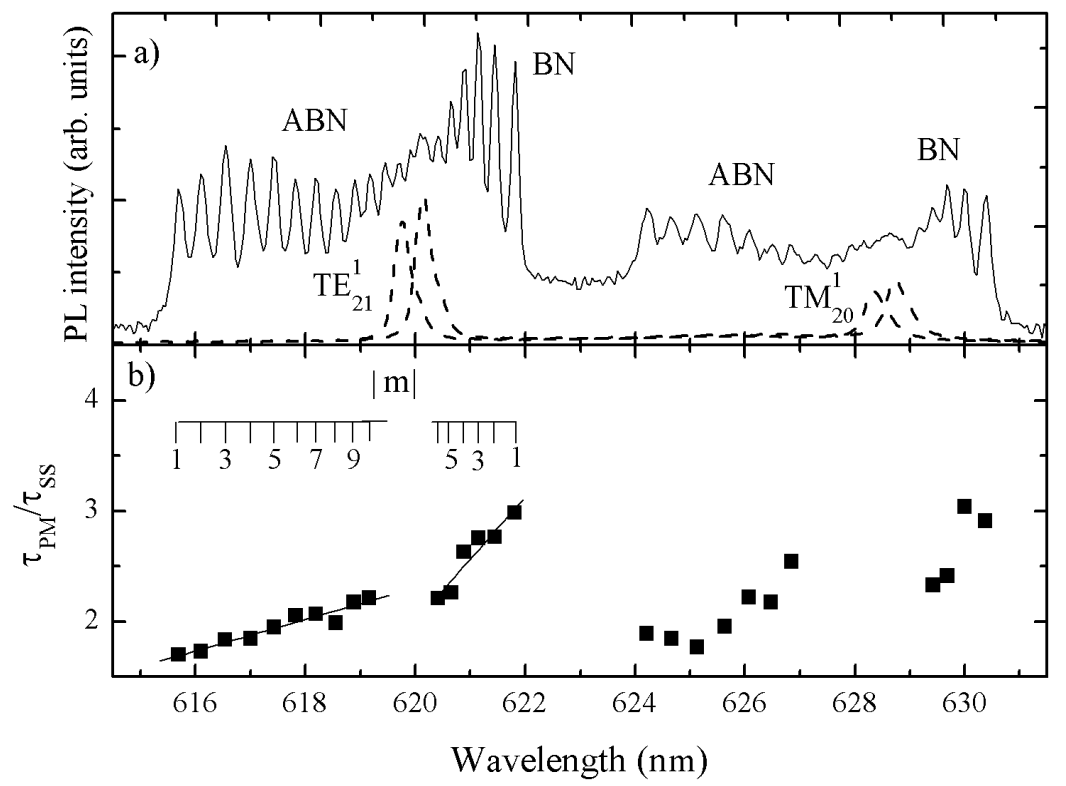

Figure 7: (a) PL spectra of the PM (solid line) formed by two almost identical melamine-formaldehyde microspheres with off-axis excitation and detection. Dashed lines show PL spectra of noninteracting microspheres. (b) Ratio between photon lifetime of the $m$-modes of photonic molecule and one of single spheres. The solid lines are result of linear fit.

The relative increase in photon storage time $R=\tau_{\mathrm{PM}} / \tau_{\mathrm{SS}}$ shows almost linear dependence on azimuthal number $m$ for given mode number $n$ (Fig.6), however it turned out that slope of this dependence $(\Delta R / \Delta m)$ in its turn varies with $n$ (Fig. 7, inset).

In our experiments we have investigated the strong coupling phenomenon in the spectra of the PM in the visible and near-IR spectral regions. In order to study the feasibility of the PM as a optical delay device, we have theoretically analysed the $m$-dependent structure for modes accommodated within the C band from 1525 - $1565 \mathrm{~nm}$ using MTA (Eq.11-13) and extrapolated the $n$-dependence of $\Delta R / \Delta m$ presented in Fig. 7 into region of higher values of angular mode number. 


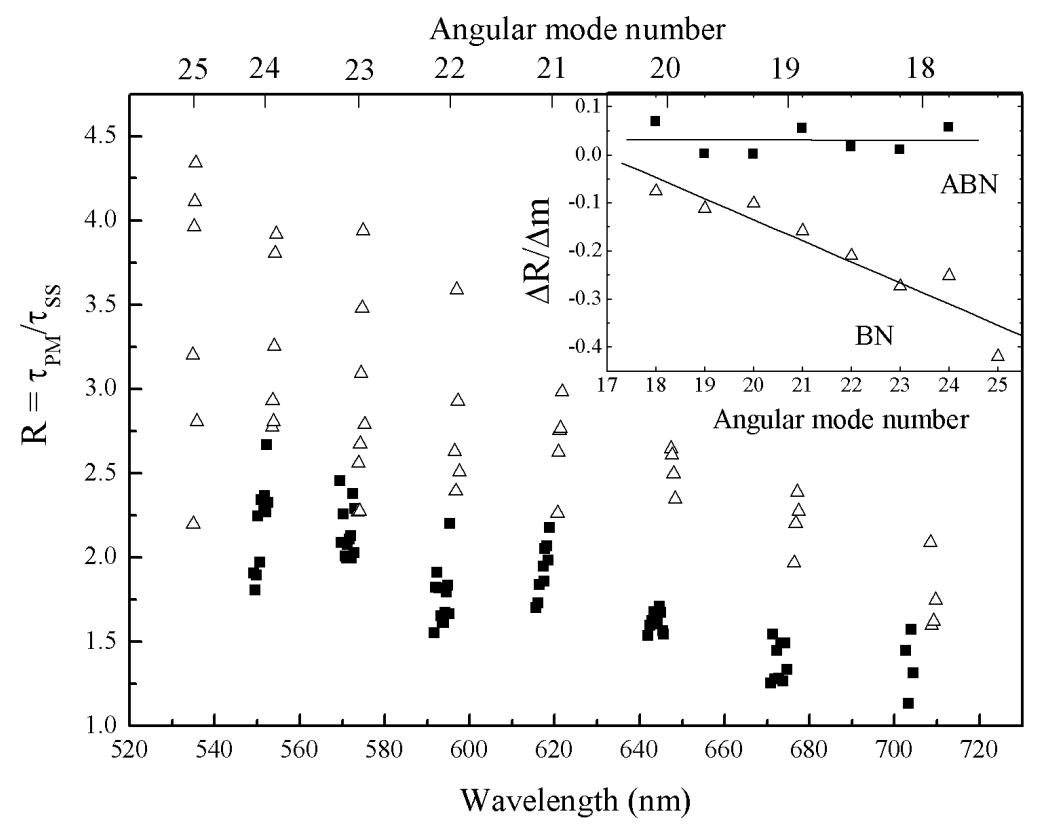

Figure 7: Ratio between photon lifetime of $m$-modes of photonic molecule $\left(\tau_{\mathrm{PM}}\right)$ and photon storage time in WGM of single sphere $\left(\tau_{\mathrm{SS}}\right)$. Inset shows $n$-dependence of slopes $\Delta \mathrm{R} / \Delta \mathrm{m}$ for $\mathrm{BN}$ and $\mathrm{ABN}$ modes. The solid lines are result of linear fit.

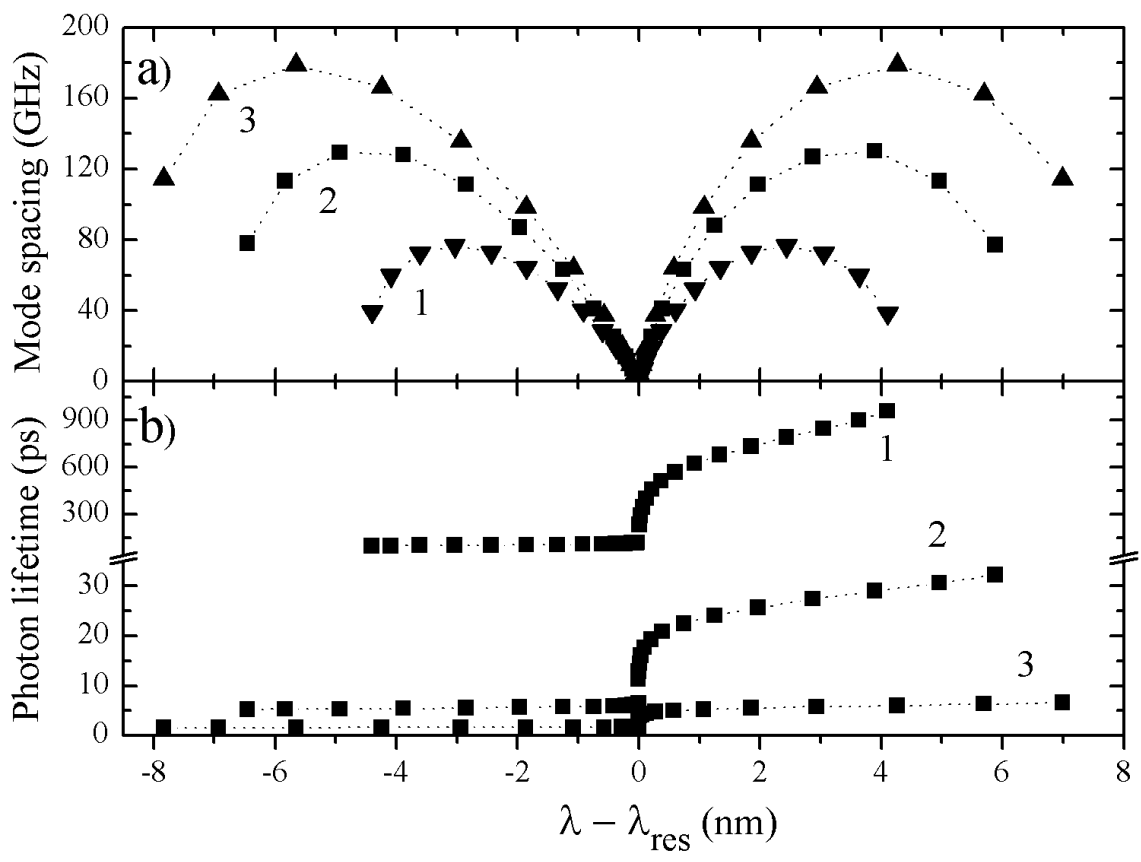

Figure 8: (a) Spacing between adjacent $m$-modes calculated in spectral region of the C-band for PMs formed by two identical $\mathrm{SiO}_{2}$ microspheres with sizes of $10 \mu \mathrm{m}$ (3), $12 \mu \mathrm{m}$ (2) and $16 \mu \mathrm{m}$ (1). (b) Calculated distribution of delay time between $m$-modes. The zero value on wavelength axis corresponds to the position of $\mathrm{TE}_{24}, \mathrm{TE}_{29}$ and $\mathrm{TE}_{40} \mathrm{WGM}$ of the single spherical microcavity.

Figure 8 a shows the values of spacing between adjacent $m$-resonances calculated in spectral region of the C-band for $\mathrm{PMs}$ formed by two identical $\mathrm{SiO}_{2}$ microspheres with sizes of $10 \mu \mathrm{m}$ (3), 12 
$\mu \mathrm{m}(2)$ and $16 \mu \mathrm{m}(1)$. The standard for channel spacing in today's optical communication systems 40 $\mathrm{GHz}-100 \mathrm{GHz}^{23}$ was taken into account deciding on microsphere sizes. In our model each $\mathrm{m}$ resonance in the PM signal can be considered as a channel with spacing between channels depending on the size of the microspheres and the azimuthal mode number.

For $10-\mu \mathrm{m}$ individual microcavities, only one TE mode $\left(\lambda_{24}=1542.4 \mathrm{~nm}\right)$ is found in the Cband. The maximum splitting between $\mathrm{BN}$ and $\mathrm{ABN}$ modes (i.e. for $m=1$ ) of $15.7 \mathrm{~nm}$ was estimated in this case. Calculating the positions of resonances for asingle $16-\mu \mathrm{m}$ microsphere we have found 2 WGMs in the region of the C-band: $\operatorname{TE}_{40}(\lambda=1544.6 \mathrm{~nm})$ and $\mathrm{TM}_{39}(\lambda=1559.6 \mathrm{~nm})$. Maximum splitting in this case is much smaller: $8.8 \mathrm{~nm}$ for the $\mathrm{TE}_{40} \mathrm{WGM}$, but accommodates a larger number of $m$ resonances. These results clearly demonstrate that two coupled WGM spherical microcavities can generate modal structure with controllable number of peaks distributed across the major communication band. In contrast to the case of single spheres, the bandpass of the proposed delay-line device can be as wide as the width of all communication bands and can be controlled by the size of spherical microcavities forming PM.

Figure $8 \mathrm{~b}$ shows the estimated distribution of delay times between m-modes of the PM taking values of the photon storage time in single microspheres to be $\tau_{\mathrm{SS}}=55 \mathrm{ps}$ for $T E_{40}^{1}, 3$ ps for $T E_{29}^{1}$ and $0.9 \mathrm{ps}$ for $T E_{24}^{1}$ as determined from our calculations based on the Mie-theory. We can anticipate that values of delay time, $\tau_{\mathrm{PM}}$, calculated in such a way are underestimated, because of the difference in Q-factor achievable for 3- $\mu \mathrm{m}$ spheres and $10-16 \mu \mathrm{m}$ ones. Recent experimental studies clearly show a strong dependence of the Q-factors of $m$-resonances (and therefore delay times) on quality factor of individual microspheres ${ }^{15,16}$.

The observed non-uniformity of spacing between $m$-resonances (Fig.7, Fig.8a) provides a unique possibility to control the distribution of photon storage times in a particular spectral region (Fig.8b). For all sizes of interacting microspheres, we have obtained an initial increase of the spacing within the region of first three $m$-resonances followed by a dip and then an increase in spacing again (Fig. 8a). This theoretical result is in good qualitative agreement with experimental data ${ }^{16}$. For the smallest PM size, $18 \mathrm{~m}$-modes are available within a window of $12-200 \mathrm{GHz}$. The smaller Q-factor of WGMs in the individual spheres forming the PM results in a relatively moderate increase in delay time - from 1.5 ps (obtained for ABN resonance with $m=1$ ) up to 7 ps calculated for the corresponding $m$ resonance of $\mathrm{BN}$ mode of PM. For microsphere of larger size, the number of available modes increases. Indeed, for $12-\mu \mathrm{m}$ coupled spheres $20 \mathrm{~m}$-resonances fit into above indicated spacing window, with biggest value of intermode spacing $\sim 130 \mathrm{GHz}$. Delay times distributed between these $m$-modes increase from 5 ps up to 32 ps. The upper limit of intermode spacing drops even more for PMs formed from $16-\mu \mathrm{m}$ microspheres. In that case, $22 \mathrm{~m}$-resonances with spacing between 12 and $76 \mathrm{GHz}$ can be seen in Fig. 8a, providing discrete time delays found to be distributed between 94 ps and 960 ps. Remarkably, this maximum delay provided by two interacting microspheres of $16-\mu \mathrm{m}$ size corresponds to a $\sim 15 \mathrm{~cm}$ length of silica-glass waveguide delay line.

\section{CONCLUSIONS}

Using these results we can draw a few general conclusions. In the weak coupling regime interacting spherical microcavities provide the possibility of waveguiding and wavelength switching in the PM depending on the excitation-detection geometry. When coupling is strong, two spherical microcavities can generate modal structure with quality factors and photon lifetimes higher than that of single spheres. By analysing the $m$-dependent splitting of $\mathrm{BN}$ and $\mathrm{ABN}$ modes of PMs as a function of size, we have introduced a new strategy for the potential application of coupled spherical microcavities as a multi-channel, wavelength-tunable optical delay device. In the proposed scheme the bandpass width, mode spacings and delays depend only on the physical parameters of the interacting microcavities and have the desired property of being independent of the modulation frequency of the communication signal.

\section{ACKNOWLEDGEMENTS}

This work was supported by Science Foundation Ireland under grant numbers 02/IN.1/I47 and 00/PI.1/C077A.2 


\section{REFERENCES}

1. J.D. Joannopoulos, P.R. Villeneuve, S. Fan, "Photonic crystals: putting a new twist on light", Nature, 386, 143-149, 1997.

2. K.J. Vahala, "Optical microcavities", Nature, 424, 839-846, 2003.

3. E. Lidorikis, M.M. Sigalas, E.N. Economou, C.M. Soukoulis, "Tight-binding parametrization for photonic band gap materials", Phys. Rev. Lett., 81, 1405-1408, 1998.

4. R.K. Chang, A.J. Campillo, Optical Processes in Microcavities, World Scientific, Singapore, 1996.

5. P. Eisberg, R. Resnick, Quantum Physics of Atoms, Molecules, Solids, Nuclei and Particles, Wiley, New York, 1985.

6. S. Arnold, J. Comunale, W.B. Whitten, J.M. Ramsey, K.A. Fuller, "Room-temperature microparticle-based persistent hole-burning spectroscopy", J. Opt. Soc. Am. B, 9, 819-824, 1992.

7. M. Bayer, T. Gutbrod, J.P. Reithmaier, A. Forchel, T.L. Reinecke, P.A. Knipp, A.A. Dremin, V.D. Kulakovskii, "Optical modes in photonic molecules", Phys. Rev. Lett., 81, 2582-2585, 1998.

8. K.A. Fuller, "Optical Resonances and Two-Sphere Systems", Appl. Opt., 30, 4716-4731, 1991.

9. T. Mukaiyama, K. Takeda, H. Miyazaki, Y. Jimba, M. Kuwata-Gonokami, "Tight-Binding Photonic Molecule Modes of Resonant Bispheres", Phys. Rev. Lett., 82, 4623-4626, 1999.

10. H. Miyazaki, Y. Jimba, "Ab initio tight-binding description of morphology-dependent resonance in a bisphere", Phys. Rev. B, 62, 7976-7997, 2000.

11. S. Arnold, A. Ghaemi, P. Hendrie, K.A. Fuller, "Morphological resonances detected from a cluster of two microspheres", Opt. Lett., 19, 156-158, 1994.

12. Y. Hara, T. Mukaiyama, K. Takeda, M. Kuwata-Gonokami, "Photonic molecule lasing", Opt. Lett., 28, 2437-2439, 2003.

13. B.M. Möller, U. Woggon, M.V. Artemyev, R. Wannemacher, "Photonic molecules doped with semiconductor nanocrystals", Phys. Rev. B, 70, 115323-1/5, 2004.

14. M.D. Barnes, S.M. Mahurin, A. Mehta, B.G. Sumpter, D.W. Noid, "Three-dimensional photonic "molecules" from sequentially attached polymer-blend microparticles", Phys. Rev. Lett., 88, 015508/1-4, 2002.

15. Y.P. Rakovich, M. Gerlach, A.L. Bradley, J.F. Donegan, T.M. Connolly, J.J. Boland, M.A. Przyjalgowski, A. Ryder, N. Gaponik, A.L. Rogach, "Confined optical modes in small photonic molecules with semiconductor nanocrystals", J. Appl. Phys., 96, 6761-6765, 2004.

16. Y.P. Rakovich, J.F. Donegan, M. Gerlach, A.L. Bradley, T.M. Connolly, J.J. Boland, N. Gaponik, A.L. Rogach, "Fine structure of coupled optical modes in photonic molecules", Phys. Rev. A, 70, 051801(R)-1/4, 2004.

17. Y.P. Rakovich, L. Yang, E.M. McCabe, J.F. Donegan, T. Perova, A. Moore, N. Gaponik, A. Rogach, "Whispering Gallery Mode Emission from a Composite System of CdTe Nanocrystals and a Spherical Microcavity", Sem. Sci. Techn., 18, 914-918, 2003.

18. C.F. Bohren, D.R. Huffman, Absorption and scattering of light by small particles, Wiley, New York, 1983.

19. P. Chýlek, "Resonance structure of Mie scattering: distance between resonances", J. Opt. Soc. Am. A, 7,1609-1613, 1990.

20. M. Kerker, The scattering of light and other electromagnetic radiation, Academic P., New York, 1969.

21. B.A. Hunter, M.A. Box, B. Maier, "Resonance structure in weakly absorbing spheres", J. Opt. Soc. Am. A, 5, 1281-1286, 1988.

22. M. Srinivasarao, D. Collings, A. Philips, S. Patel, "Three-dimensionally ordered array of air bubbles in a polymer film", Science, 292, 79-83, 2001.

23. A. Gumaste, T. Antony, DWDM Network Designs and Engineering Solutions, Cisco Press, Indianapolis, 2002. 